\title{
New sources of sorghum resistant genotypes to downy mildew disease in Uganda
}

\author{
FRANK KUMI $^{1,2, \bullet}$, ARFANG BADJI ${ }^{1}$, NATASHA MWILA ${ }^{1}$, THOMAS ODONG ${ }^{1}$, MILDRED OCHWO-

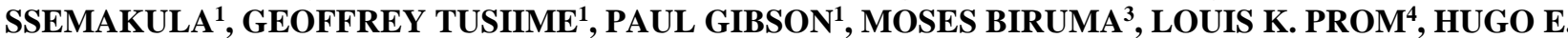 \\ CUEVAS $^{5}$, SYMPHORIEN AGBAHOUNGBA ${ }^{6}$, PATRICK RUBAIHAYO ${ }^{1}$ \\ ${ }^{1}$ Department of Agricultural Production, College of Agricultural and Environmental Sciences, Makerere University. P.O. Box 7062, Kampala, Uganda \\ Tel.: +256-414-542277, `email: frankkumifk@gmail.com \\ ${ }^{2}$ Department of Crop Science, College of Agricultural and Natural Sciences, University of Cape Coast, P.M.B Cape Coast, Ghana. \\ ${ }^{3}$ National Semi-Arid Resources Research Institute (NaSARRI), Serere, Uganda \\ ${ }^{4}$ USDA-ARS, Southern Plains Agriculture Research Center, College Station, Texas, United States of America \\ ${ }^{5}$ USDA-ARS, Tropical Agriculture Research Station, Mayaguez, Puerto Rico \\ ${ }^{6}$ Laboratory of Applied Ecology, University of Abomey-Calavi, Cotonou, Benin
}

Manuscript received: 28 August 2019. Revision accepted: 29 October 2019.

\begin{abstract}
Kumi F, Badji A, Mwila N, Odong T, Ochwo-Ssemakula M, Tusiime G, Gibson P, Biruma M, Prom KL, Cuevas HE, Agbahoungba S, Rubaihayo P. 2019. New sources of sorghum resistant genotypes to downy mildew disease in Uganda. Biodiversitas 20: 3391-3397. Sorghum downy mildew (SDM) disease is still prevalent in Uganda at varying levels of incidence and severity. In this study, a total of 100 sorghum genotypes, five (5) from (U.SA, India, and Sudan) and 95 genotypes from Uganda were evaluated for resistance to downy mildew and other agronomic traits during the second growing season of 2016 (August-December). The experiment was conducted in two locations at Makerere University Agricultural Research Institute at Kabanyolo (MUARIK) and Abi-Zonal Agricultural Research and Development Institute (Abi-ZARDI) research station at Arua. The experimental design used was 10 x 10 alpha lattice design with three replicates. Data were collected on plant disease incidence (PDI), plant disease severity (PDS), area under disease progress curve (AUDPC), days to 50\% flowering, plant height, 1000 seed weight, and grain yield. Results for analysis of variance showed highly significant differences $(\mathrm{P}<0.001)$ in genotypes, location, and AUDPC, yield and yield components. Disease incidence varied significantly $(\mathrm{P}<0.001$ ) between locations, and Arua recorded highest disease incidence and severity of 80.6 and 2.8 , respectively. Results from correlation analysis showed a highly significant $(\mathrm{P}<0.001)$ positive association of downy mildew disease incidence with AUDPC (0.835) which suggests that the severity of SDM disease increased with disease incidence, whiles significant (P $<0.001)$ negative correlation was recorded for days to $50 \%$ flowering $(-0.302), 1000$ seed weight $(-0.471)$, and grain yield (-0.585), suggesting that grain yield and yield component decreased significantly with increase in SDM incidence and severity. Two resistant (PI 656061 and PI 533831) and four moderately resistant (E 40, MAKSO 8, PI 655990 and Epuripur) genotypes were identified from this study. These genotypes were recommended for sorghum breeding program against downy mildew disease.
\end{abstract}

Keywords: Angle transformation, AUDPC, PDI, local lesions, systemic infection

\section{INTRODUCTION}

Sorghum (Sorghum bicolor (L) Moench) is an important cereal crop grown mainly on subsistence basis by farmers in Uganda (Wasake 2016). It is the fifth most important staple food crop after banana, cassava, maize, and millet, occupying over 400,000 hectares of arable land (FAO 2013). Besides the consumption and industrial uses of sorghum grain, the crop residues and green plants also provide sources of animal feed, building material, fuel for cooking, particularly in dryland areas. It is agronomically suited to hot and dryland growing areas in a temperature range of 12-37 $\mathrm{o}$ - where other cereal crops will fail to thrive or yield substantially (Rao and Kumar 2013). The production of sorghum is challenged by several diseases of which sorghum downy mildew (SDM) is one of the most destructive which greatly reduces the productivity of the crop (Frederiksen 2000) and is reported to be endemic in Uganda (Kumi et al.2018a).
Sorghum downy mildew is caused by Peronosclerospora sorghi which is a serious disease of sorghum and other host plants such as maize, sugar cane, Sudan grass and Johnson grass (Bigirwa et al. 2000; Shaarawy et al. 2002). Affected plants show both systemic symptoms and local lesions at varying severity levels depending on the cultivar and occurs at any stage of development under favorable environmental conditions (Pande et al. 1997). Systemic infections may arise from soil-borne oospores that are sexually reproduced (Tuleen et al. 1980) and serves as primary inoculum source, or can aerially be dispersed through asexually formed conidia structure. Local and systemic lesions significantly contribute to yield loss can act as a source of conidial inoculum for subsequent systemic infection in younger susceptible plants (Cohen and Sherman 1977). Chemical control of SDM disease does not only pose economic strain on resourced poor farmers in sub-Saharan Africa but it also degrades the environment. Metalaxyl resistance of $P$. sorghi pathotypes has been reported in areas where 
extensive fungicide application has been deployed (Isakeit et al. 2003). These observations, coupled with emergence of $P$. sorghi pathotypes make the need for genetic resistance through breeding to be more efficient, sustainable, environmentally friendly and cost-effective management approach for SDM control (Radwan et al. 2011). Numerous resistant lines of sorghum to SDM disease have been identified in countries other than Uganda (Miller et al. 1992; Prom et al. 2007), and reports of screening sorghum and maize germplasm against SDM disease are available in India (Kamala et al. 2006), China (Prom et al. 2007), Mexico and USA (Prom et al. 2014) and Brazil (Barbosa et al. 2004).

Up to date, no resistant SDM cultivar has been released in Uganda nor a study carried out to identify sources of resistance to SDM disease. Therefore, this study sought to screen a total of 100 sorghum genotypes including five (5) sorghum introductions from India (P1 656061), Sudan (P1 533831) and USA (P1 655990, P1 669760, and P1 656010) with the aim of identifying sources of sorghum resistance to downy mildew disease with better agronomic traits under natural infection at two different locations, Makerere University Agricultural Research Institute at Kabanyolo (MUARIK) and Abi-Zonal Agricultural Research and Development Institute (Abi-ZARDI) research station at Arua.

\section{MATERIALS AND METHODS}

\section{Materials}

A total of 100 sorghum genotypes were used in this experiment. These genotypes comprised of 5 introductions including two resistant checks PI 656061 (QL3-India) and P1 533931 (Sudan) sourced from the Gene-Bank of United States Department of Agriculture (USDA-ARS), 66 landraces and 29 improved cultivars all sourced from the National Semi-Arid Resources Research Institute (NaSARRI) Serere, Uganda. Seed multiplication was done for the 5 inbred lines sourced from USDA in the Screen house at the Makerere University Agricultural Research Institute Kabanyolo (MUARIK) to general appreciable seed quantity for the experiment.

\section{Experimental sites}

Evaluations of the 100 genotypes were done at two locations namely, MUARIK located at $0^{\circ} 28^{\prime} 57^{\prime \prime} \mathrm{N}$; $32^{\circ} 35^{\prime} 42.0^{\prime \prime} \mathrm{E}(1152 \mathrm{~m}$ above sea level) and Abi-ZARDI located at $3^{\circ} 04^{\prime} 18^{\prime \prime} \mathrm{N} ; 30^{\circ} 54^{\prime} 39^{\prime \prime} \mathrm{E}$ (1202 m above sea level) during the second rainy season (August-November) of 2016. The temperature range, average rainfall and relative humidity recorded during the experimental period were 19$28^{\circ} \mathrm{C}, 101 \mathrm{~mm}$, and $55-88 \%$, respectively for MUARIK and $20-30^{\circ} \mathrm{C}, 87 \mathrm{~mm}$, and $60-71 \%$, respectively for AbiZARDI.

\section{Experimental design}

An alpha lattice experimental design of $10 \times 10$ with 3 replicates were used at each site. Each replicates had a total of 10 blocks, and each block had 10 plots. Each sorghum genotype was planted on a $4 \mathrm{~m}$ row with a planting distance of $60 \mathrm{~cm}$ as inter-row spacing and $30 \mathrm{~cm}$ as the intra-row spacing. Inter plot distance was $1 \mathrm{~m}$ and $2 \mathrm{~m}$ distance was used as the spacing between replications. The sowing rate was 3 seeds per hole and thinned to 2 plants per stand after full sprouting (after 2 weeks of emergence). Weeding was manually done at different periods until harvesting.

\section{Data collection and analyses}

Five weeks after planting, five plants were randomly selected from each experimental plot, tagged and visually evaluated for SDM incidence and severity. The evaluation was done at seven days interval for six successive more weeks purposefully for area under disease progress curve (AUDPC) estimation. Disease incidence was done 78 days after planting using key SDM symptoms such as leaf streaks, necrotic leaf, chlorosis, downy appearance, stunted growth and vein clearing (Jeger et al. 1998) by randomly assessing five sorghum plants on each plot. Percentage disease incidence (PDI) was then estimated from the disease incidence score by counting the number of plants showing SDM symptoms in each plot as against the total number of plants in each plot using Ward's formula (Ward 1997):

$$
\text { PDI }=\frac{\text { Number of plants infected }}{\text { Total number of plants }} x 100
$$

PDI data was angular transformed (Williams et al. 1990) to normalize the data (Sokal and Rohlf 1995) before ANOVA was performed in Genstat 12.0 software (Payne et al. 2009). Disease severity readings were recorded for seven consecutive times from each experimental plot. The severity scoring was done using a severity scale of 1-5 adopted from Ward (1997):

Where,

1 : No symptoms on leaves,

$2: 1-25 \%$ leaf area infection,

$3: 26-50 \%$ leaf area infection,

$4: 51-75 \%$

$5: 76-100 \%$ leaf area infection.

Means were subjected to Fisher's Least Significant Difference (Lsd) tests at a probability level of 5\%. Mean severity scores generated from the ANOVA results were used to estimate the (AUDPC) for each genotype using Microsoft Excel 2007 Office suite. The formula used to estimate AUDPC (Simko and Piepho 2012):

$$
A U D P C=\sum_{i=1}^{n-1}\left(\frac{y_{i}+y_{i+1}}{2}\right) x\left(t_{i+1}-t_{i}\right)
$$

Where, $y_{i}$ is an assessment of a disease severity score at the $i^{\text {th }}$ observation, $t_{i}$ is the time (days) at the $i^{\text {th }}$ observation, and $n$ is the total number of observations.

Mean incidence data for each genotype was further used as a modified qualitative classification scale adopted from Bock et al. (2000) to classify each genotype response 
to SDM infection. The scale used was; <20\% plant infection was classified resistant, $20-50 \%$ plant infection was classified moderately resistant, and > 50\% plant infection was susceptible.

Data on agronomic traits such as days to $50 \%$ flowering for each genotype, 1000 seed weight $(\mathrm{g})$, plant height $(\mathrm{cm})$ were recorded and grain yield ( $\mathrm{t} / \mathrm{ha}$ ) was estimated per plot. Plot means for yield, yield components and SDM disease incidence and severity values were subjected to One-way analysis of variance (ANOVA) in Genstat version 12.0 software (Payne et al. 2009) and Fisher's LSD test was used for mean separation. Pearson correlation was done in Genstat 12.0 software (Payne et al. 2009) among agronomic traits and SDM disease parameters (PDI and AUDPC) to examine any association between agronomic traits and SDM disease parameters.

\section{RESULTS AND DISCUSSION}

\section{Variability of genotypes to downy mildew disease}

The results of analysis of variance for the downy mildew disease incidence (PDI), disease severity (PDS), AUDPC and agronomic traits (days to $50 \%$ flowering, plant height, 1000 seed weight and grain yield) evaluated at Abi-ZARDI and MUARIK are presented in Table 1. The results showed highly significant differences $(\mathrm{P}<0.001)$ among the 100 genotypes for PDI, PDS, AUDPC, days to $50 \%$ flowering, plant height, 1000 seed weight and grain yield. Similarly, highly significant differences $(P<0.001)$ were observed between location for PDI, PDS, AUDPC, days to $50 \%$ flowering, plant height, 1000 seed weight, and grain yield. Genotype $\mathrm{x}$ location interactions recorded highly significant differences for downy mildew parameters PDI, PDS, and AUDPC but no interaction effect was recorded for agronomic trait days to $50 \%$ flowering, plant height, 1000 seed weight and grain yield. These variations could be attributed to genetic differences among the genotypes and the interaction effects are primarily due to the observed significant differences in disease pressure at both locations. Kamala et al. (2002) and Prom et al. (2010, 2014) reported similar variation among sorghum genotypes response to SDM disease.

\section{Variability of SDM disease parameters to sorghum yield at different locations}

The results for mean values of downy mildew disease incidence (PDI), disease severity (PDS), disease reaction of genotype, area under disease progress curve (AUDPC) and yield, recorded at Abi-ZARDI and MUARIK are presented in Table 2. The results showed a higher disease incidence, severity and AUDPC mean values at Abi-ZARDI in the West Nile Region and lower disease incidence, severity, and AUDPC mean values for at MUARIK in the Central Region. These results confirmed that Abi-ZARDI in Arua was a hotspot for downy mildew disease (Kumi et al. 2018a).

Downy mildew disease incidence at Abi-ZARDI ranged between $6.7-100 \%$ with a mean incidence of $80.6 \%$. MUARIK recorded disease incidence ranged between 0$60 \%$ with a mean incidence of $30.1 \%$. Disease severity for downy mildew at Abi-ZARDI and MUARIK ranged between 1.1-3.5 and 1.0-2.1, respectively. The mean severity recorded for Abi-ZARDI and MUARIK was 2.8 and 1.6, respectively. The results showed a high disease pressure at Abi-ZARDI compared to relatively lower disease pressure at MUARIK (Table 2). Reaction of genotypes to downy mildew infection under natural infection yielded varying results for SDM resistance ratings based on PDI values at the two locations. Out of the 100 genotypes screened, 10 genotypes (1\%) (E 40, ENT 183, MAKSO 1, MAKSO 5, MAKSO 8, P 3, P 6, PI 533831 (Sudan), PI 656061 (QL3-India), W 3) were found to be resistant. Two of these genotypes, PI 533831 (Sudan) and PI 656061 (QL3-India) were found to be resistant (PDI < $10 \%$ ) at both Abi-ZARDI and MUARIK. Conversely, six genotypes ENT 40, MAKSO 1, MAKSO 5, P 3, P 6 and W 2 which were found to be resistant to downy mildew at MUARIK were susceptible (PDI > $50 \%$ ) at Abi-ZARDI confirming high downy mildew disease pressure AbiZARDI in Arua (Kumi et al. 2018a). Bigirwa et al. (1998) reported variations of downy disease incidence within different locations in Uganda. Significant variations for genotype response to downy mildew disease at the two locations in this study could be attributed to differences in disease pressure as influenced by the different environments (Aegerter et al. 2003). Mean AUDPC value recorded in Abi-ZARDI (65.1) was higher compared with the values in MUARIK (51.3) (Table 2). Sorghum yield recorded in this study was generally high at MUARIK compared to the yield at Arua (Table 2). The mean yield recorded at MUARIK ranged between $0.92 \mathrm{t} / \mathrm{ha}(\mathrm{P} 20)$ to 2.32 t/ha PI 656061 (QL3-India), whiles the mean yield at Abi-ZARDI ranged between $0.55 \mathrm{t} / \mathrm{ha}$ (E 2) to $1.91 \mathrm{t} / \mathrm{ha} \mathrm{PI}$ 656061 (QL3-India). Since the same genotypes were evaluated at both locations, the observed significant difference can be attributed to the effect of downy mildew disease pressure (PDI and AUDPC values) and possible occurrence of $P$. sorghi pathotypes (Kumi et al. 2018b). Low sorghum yield recorded at Abi-ZARDI was due to high disease pressure at the location compared to high yield in MUARIK and this finding corroborated to the linear association for downy mildew disease impact on yield decline/loss (Wall et al. 1992).

\section{Association between downy mildew disease parameters and agronomic traits}

The extent to which the studied traits contributed to increase resistance to downy mildew disease were obtained through the results of correlation coefficient (r) presented in Table 3. 
Table 1. Combined mean sum of square for downy mildew disease incidence, severity, AUDPC and agronomic traits of 100 sorghum genotypes at Arua and Kabanyolo

\begin{tabular}{|c|c|c|c|c|c|c|c|c|}
\hline SOV & df & PDI & PDS & AUDPC & $\begin{array}{c}\text { Days to 50\% } \\
\text { flowering } \\
\text { (days) }\end{array}$ & $\begin{array}{l}\text { Plant height } \\
(\mathbf{c m})\end{array}$ & $\begin{array}{c}1000 \text { seed } \\
\text { weight }(\mathrm{gm})\end{array}$ & $\begin{array}{l}\text { Grain } \\
\text { yield } \\
\text { (t/ha) }\end{array}$ \\
\hline Genotype & 99 & $979.9 * * *$ & $0.411^{* * *}$ & $118.933 * * *$ & $70.652 * * *$ & $159106.80^{* * *}$ & $25.678 * * *$ & $0.381 * * *$ \\
\hline Location & 1 & $383548.3 * * *$ & $200.219 * * *$ & $28659.208^{* * *}$ & $2156.473 * * *$ & $31637.30^{* * *}$ & $1060.269 * * *$ & $53.908 * * *$ \\
\hline $\mathrm{G} \times \mathrm{L}$ & 99 & $568.7 * *$ & $0.2656^{* *}$ & $76.272 * * *$ & $0.974^{\mathrm{NS}}$ & $370.70^{\mathrm{NS}}$ & $0.099^{\mathrm{NS}}$ & $0.045^{\mathrm{NS}}$ \\
\hline Residual & 400 & 376.2 & 0.1615 & 4.168 & 2.436 & 861.30 & 1.337 & 0.100 \\
\hline
\end{tabular}

Table 2. Mean values for PDI, PDS, AUDPC, Rating, and Yield recorded for 100 sorghum genotypes evaluated in 2016 at Abi-ZARDI and MUARIK, Uganda

\begin{tabular}{|c|c|c|c|c|c|c|c|c|c|c|}
\hline \multirow[b]{2}{*}{ Genotype } & \multicolumn{5}{|c|}{ Abi-ZARDI } & \multicolumn{5}{|c|}{ MUARIK } \\
\hline & PDI $^{\mathbf{a}}(\%)$ & Rating & $\mathbf{P D S}^{\mathbf{b}}$ & AUDPC & $\begin{array}{l}\text { Yield } \\
\text { (t/ha) }\end{array}$ & $\mathbf{P D I}^{\mathbf{a}}(\%)$ & Rating & $\mathbf{P D S}^{\mathbf{b}}$ & AUDPC & $\begin{array}{l}\text { Yield } \\
\text { (t/ha) }\end{array}$ \\
\hline AG 8 & $93.3(75.0)$ & $\mathrm{S}$ & 3.2 & 72.44 & 0.74 & $46.7(43.1)$ & MR & 1.9 & 56.82 & 1.74 \\
\hline B 3 & $80.0(63.4)$ & S & 2.6 & 62.61 & 0.88 & $26.7(31.1)$ & MR & 1.7 & 53.18 & 1.43 \\
\hline E 10 & 86.7 (68.6) & $\mathrm{S}$ & 3.2 & 72.44 & 1.16 & $26.7(31.1)$ & MR & 1.4 & 47.72 & 1.89 \\
\hline E 12 & 86.7 (68.6) & $\mathrm{S}$ & 2.9 & 67.52 & 0.89 & $46.7(43.1)$ & MR & 1.6 & 51.36 & 1.45 \\
\hline E 16 & $93.3(75.0)$ & $\mathrm{S}$ & 3.1 & 70.80 & 0.82 & $33.3(35.2)$ & MR & 1.7 & 53.18 & 1.46 \\
\hline E 19 & $100.0(84.7)$ & $S$ & 3.1 & 70.80 & 0.84 & $20.0(26.6)$ & MR & 1.4 & 47.72 & 1.56 \\
\hline E 2 & $100.0(84.7)$ & S & 3.5 & 77.35 & 0.55 & $46.7(43.1)$ & MR & 1.9 & 56.82 & 1.47 \\
\hline E 20 & 86.7 (68.6) & S & 2.7 & 64.25 & 0.70 & $20.0(26.6)$ & MR & 1.5 & 49.54 & 1.13 \\
\hline E 22 & $93.3(75.0)$ & S & 2.8 & 65.88 & 0.77 & $53.3(46.9)$ & $\mathrm{S}$ & 1.9 & 56.82 & 1.25 \\
\hline E 23 & $86.7(68.6)$ & $S$ & 2.7 & 64.25 & 0.70 & $46.7(43.1)$ & MR & 1.9 & 56.82 & 1.13 \\
\hline E 3 & $80.0(63.4)$ & $S$ & 2.9 & 67.52 & 0.72 & $46.7(43.1)$ & MR & 1.9 & 56.82 & 1.18 \\
\hline E 4 & $100.0(84.7)$ & $S$ & 3.1 & 70.80 & 0.74 & $40.0(39.2)$ & MR & 1.8 & 55.00 & 1.39 \\
\hline E 40 & $26.7(31.1)$ & MR & 1.6 & 46.23 & 1.16 & $13.3(21.4)$ & $\mathrm{R}$ & 1.2 & 44.08 & 1.89 \\
\hline E 44 & $80.0(63.4)$ & $\mathrm{S}$ & 2.8 & 65.88 & 0.72 & $53.3(46.9)$ & S & 1.7 & 53.18 & 1.18 \\
\hline E 5 & $100.0(84.7)$ & $S$ & 3.2 & 72.44 & 0.84 & $53.3(46.9)$ & $S$ & 1.7 & 53.18 & 1.64 \\
\hline E 6 & $93.3(75.0)$ & $S$ & 3.1 & 70.80 & 0.73 & $33.3(35.2)$ & MR & 1.8 & 55.00 & 1.57 \\
\hline E 60 & $100.0(84.7)$ & $S$ & 3.1 & 70.80 & 0.78 & $33.3(35.2)$ & MR & 1.5 & 49.54 & 1.74 \\
\hline E 7 & $100.0(84.7)$ & $S$ & 3.3 & 74.04 & 0.66 & $46.7(43.1)$ & MR & 1.7 & 53.18 & 1.39 \\
\hline E 8 & $80.0(63.4)$ & $S$ & 2.9 & 67.52 & 1.04 & $40.0(39.2)$ & MR & 1.7 & 53.18 & 1.70 \\
\hline E 9 & $53.3(46.9)$ & $S$ & 2.3 & 57.69 & 1.11 & $33.3(35.2)$ & MR & 1.5 & 49.54 & 1.80 \\
\hline E $90-1$ & $86.7(68.6)$ & S & 2.7 & 64.25 & 1.08 & $33.3(35.2)$ & MR & 1.5 & 49.54 & 1.76 \\
\hline E $90-2$ & $76.7(61.1)$ & $S$ & 2.5 & 47.72 & 1.03 & $26.7(31.1)$ & MR & 1.4 & 47.72 & 1.68 \\
\hline ENT 114 & $100.0(84.7)$ & $S$ & 3.2 & 72.44 & 0.70 & $33.3(35.2)$ & MR & 1.6 & 51.36 & 1.14 \\
\hline ENT 115 & $100.0(84.7)$ & $\mathrm{S}$ & 3.2 & 72.44 & 0.70 & $46.7(43.1)$ & MR & 1.7 & 53.18 & 1.25 \\
\hline ENT 13 & $100.0(84.7)$ & S & 3.4 & 75.71 & 0.61 & $60.0(50.8)$ & $\mathrm{S}$ & 1.8 & 55.00 & 1.46 \\
\hline ENT 130 & $100.0(84.7)$ & S & 3.1 & 70.80 & 0.79 & $33.3(35.2)$ & MR & 1.7 & 53.18 & 1.46 \\
\hline ENT 14 & $83.3(65.9)$ & S & 2.7 & 64.25 & 0.76 & $40.0(39.2)$ & MR & 1.6 & 51.36 & 1.25 \\
\hline ENT 15 & $100.0(84.7)$ & $S$ & 3.1 & 70.80 & 0.72 & $20.0(26.6)$ & MR & 1.5 & 49.54 & 1.37 \\
\hline ENT 16 & $100.0(84.7)$ & $S$ & 3.3 & 74.07 & 0.70 & $20.0(26.6)$ & MR & 1.4 & 47.72 & 1.74 \\
\hline ENT 162 & $60.0(50.8)$ & S & 2.3 & 57.69 & 0.95 & $26.7(31.1)$ & MR & 1.7 & 53.18 & 1.39 \\
\hline ENT 17 & $80.0(63.4)$ & S & 3.0 & 69.19 & 0.89 & $33.3(35.2)$ & MR & 1.5 & 49.54 & 1.45 \\
\hline ENT 173 & $100.0(84.7)$ & S & 3.2 & 72.44 & 0.87 & $26.7(31.1)$ & MR & 1.7 & 53.18 & 1.76 \\
\hline ENT 18 & $93.3(75.0)$ & $S$ & 3.1 & 70.80 & 0.80 & $26.7(31.1)$ & MR & 1.4 & 47.72 & 1.46 \\
\hline ENT 183 & $60.0(50.8)$ & S & 2.5 & 60.97 & 1.03 & $13.3(21.4)$ & $\mathrm{R}$ & 1.4 & 47.72 & 1.67 \\
\hline ENT 187 & $100.0(84.7)$ & $S$ & 3.3 & 74.07 & 0.66 & $20.0(26.6)$ & MR & 1.4 & 47.72 & 1.53 \\
\hline ENT 19 & $53.3(46.9)$ & $S$ & 2.3 & 57.69 & 1.00 & $20.0(26.6)$ & MR & 1.4 & 47.72 & 1.62 \\
\hline ENT 32 & $100.0(84.7)$ & $S$ & 3.1 & 70.80 & 0.78 & $20.0(26.6)$ & MR & 1.4 & 47.72 & 1.27 \\
\hline ENT 46 & $83.3(65.9)$ & $S$ & 2.5 & 60.97 & 1.01 & $20.0(26.6)$ & MR & 1.5 & 49.52 & 1.65 \\
\hline ENT 47 & $80.0(63.4)$ & S & 2.7 & 64.25 & 0.87 & $20.0(26.6)$ & MR & 1.3 & 45.90 & 1.42 \\
\hline ENT 48 & $86.7(68.6))$ & $S$ & 2.7 & 64.25 & 1.03 & $20.0(26.6)$ & MR & 1.5 & 49.52 & 1.68 \\
\hline ENT 49 & $100.0(84.7)$ & $S$ & 3.0 & 69.19 & 0.82 & $33.3(35.2)$ & MR & 1.8 & 55.00 & 1.39 \\
\hline ENT 50 & $80.0(63.4)$ & $S$ & 2.7 & 64.25 & 0.89 & $20.0(26.6)$ & MR & 1.5 & 49.52 & 1.45 \\
\hline ENT 97 & $100.0(84.7)$ & $S$ & 3.1 & 70.80 & 0.90 & $20.0(26.6)$ & MR & 1.4 & 47.72 & 1.46 \\
\hline $\begin{array}{l}\text { Epuripur } \\
(2 \mathrm{~K} \times 17 / \mathrm{B} / 1)\end{array}$ & $46.7(43.1)$ & MR & 2.2 & 56.06 & 1.50 & $20.0(26.6)$ & MR & 1.5 & 49.52 & 1.98 \\
\hline
\end{tabular}




\begin{tabular}{|c|c|c|c|c|c|c|c|c|c|c|}
\hline F 14a & $93.3(75.0)$ & $S$ & 3.0 & 69.16 & 0.81 & $20.0(26.6)$ & MR & 1.5 & 49.52 & 1.37 \\
\hline F 15a & $100.0(84.7)$ & $\mathrm{S}$ & 3.3 & 74.07 & 0.73 & $20.0(26.6)$ & MR & 1.7 & 53.18 & 1.45 \\
\hline MAKSO 1 & $66.7(54.8)$ & $S$ & 2.2 & 56.06 & 1.08 & $13.3(21.4)$ & $\mathrm{R}$ & 1.3 & 45.90 & 1.76 \\
\hline MAKSO 13 & $53.3(46.9)$ & S & 2.3 & 57.69 & 0.71 & $40.0(39.2)$ & MR & 1.9 & 56.82 & 1.15 \\
\hline MAKSO 14 & $93.3(75.0)$ & $\mathrm{S}$ & 3.2 & 72.44 & 0.69 & $33.3(35.2)$ & MR & 1.8 & 55.00 & 1.46 \\
\hline MAKSO 18 & $93.3(75.0)$ & S & 3.1 & 70.80 & 0.74 & $53.3(46.9)$ & S & 2.1 & 60.46 & 1.67 \\
\hline MAKSO 2 & $73.3(58.9)$ & S & 2.5 & 60.97 & 0.94 & $53.3(46.9)$ & S & 2.1 & 60.46 & 1.53 \\
\hline MAKSO 28 & 86.7 (68.6) & S & 3.1 & 70.80 & 0.76 & $26.7(31.1)$ & MR & 1.7 & 53.18 & 1.86 \\
\hline MAKSO 29 & $73.3(58.9)$ & $\mathrm{S}$ & 2.7 & 64.25 & 0.71 & $20.0(26.6)$ & MR & 1.4 & 47.72 & 1.15 \\
\hline MAKSO 30 & $93.3(75.0)$ & $\mathrm{S}$ & 2.9 & 67.52 & 0.64 & $33.3(35.2)$ & MR & 1.8 & 55.00 & 1.05 \\
\hline MAKSO 4 & $73.3(58.9)$ & S & 2.5 & 60.97 & 0.96 & $53.3(46.9)$ & $S$ & 1.9 & 56.82 & 1.57 \\
\hline MAKSO 5 & $66.7(54.8)$ & S & 2.7 & 64.25 & 0.89 & $13.3(21.4)$ & $\mathrm{R}$ & 1.3 & 45.90 & 1.45 \\
\hline MAKSO 62 & $60.0(50.8)$ & S & 2.6 & 62.61 & 0.63 & $53.3(46.9)$ & $\mathrm{S}$ & 1.9 & 56.82 & 1.03 \\
\hline MAKSO 62S & $93.3(75.0)$ & S & 2.9 & 67.52 & 0.87 & $33.3(35.2)$ & MR & 1.6 & 51.36 & 1.42 \\
\hline MAKSO 7 & $66.7(54.8)$ & $S$ & 2.3 & 57.69 & 0.89 & $40.0(39.2)$ & MR & 1.7 & 53.18 & 1.45 \\
\hline MAKSO 8 & $46.7(43.1)$ & MR & 2.3 & 57.69 & 1.01 & $13.3(21.9)$ & $\mathrm{R}$ & 1.3 & 45.90 & 1.64 \\
\hline MAKSO 9 & $93.3(75.0)$ & $\mathrm{S}$ & 3.0 & 69.16 & 1.15 & $46.7(43.1)$ & MR & 1.9 & 56.82 & 1.88 \\
\hline MUK 11 & $100.0(84.7)$ & S & 3.2 & 72.44 & 1.40 & $20.0(26.6)$ & MR & 1.5 & 49.54 & 1.93 \\
\hline MUK 128 & $93.3(75.0)$ & S & 3.1 & 70.80 & 0.60 & $20.0(26.6)$ & MR & 1.5 & 49.54 & 1.64 \\
\hline MUK 129 & 86.7 (68.6) & $\mathrm{S}$ & 2.9 & 67.52 & 1.04 & $26.7(31.1)$ & MR & 1.6 & 51.36 & 1.69 \\
\hline MUK 154 & $66.7(54.8)$ & $S$ & 2.7 & 64.25 & 1.01 & $26.7(31.1)$ & MR & 1.4 & 47.72 & 1.65 \\
\hline MUK 155 & $66.7(54.8)$ & S & 2.5 & 60.97 & 1.08 & $20.0(26.6)$ & MR & 1.3 & 45.90 & 1.76 \\
\hline MUK 159 & $60.0(50.8)$ & S & 2.1 & 54.42 & 0.93 & $20.0(26.6)$ & MR & 1.3 & 45.90 & 1.51 \\
\hline MUK 27 & $100.0(84.7)$ & S & 2.9 & 67.52 & 1.34 & $26.7(31.1)$ & MR & 1.6 & 51.36 & 1.65 \\
\hline MUK 33 & $73.3(58.9)$ & $\mathrm{S}$ & 2.7 & 64.25 & 1.62 & $33.3(35.2)$ & MR & 1.8 & 55.00 & 1.87 \\
\hline MUK 51 & $80.0(63.4)$ & S & 2.6 & 62.61 & 1.07 & $20.0(26.6)$ & MR & 1.3 & 45.90 & 1.75 \\
\hline MUK 59 & $86.7(68.6)$ & $S$ & 2.7 & 64.25 & 1.27 & $20.0(26.6)$ & MR & 1.5 & 49.54 & 2.08 \\
\hline MUK 60 & $66.7(54.8)$ & $S$ & 2.6 & 62.61 & 0.71 & $20.0(26.6)$ & MR & 1.3 & 45.90 & 1.15 \\
\hline P 1 & 86.7 (68.6) & S & 2.6 & 62.61 & 1.01 & $26.7(31.1)$ & MR & 1.5 & 49.54 & 1.64 \\
\hline P 10 & 86.7 (68.6) & S & 2.5 & 60.97 & 0.89 & $46.7(43.1)$ & MR & 1.9 & 56.54 & 1.44 \\
\hline P 13 & $73.3(58.9)$ & S & 2.3 & 57.69 & 0.72 & $40.0(39.2)$ & MR & 2.0 & 58.64 & 1.17 \\
\hline P 14 & $93.3(75.0)$ & $S$ & 2.7 & 64.25 & 0.76 & $40.0(39.2)$ & MR & 1.9 & 56.54 & 1.24 \\
\hline P 15 & $80.0(63.4)$ & $S$ & 2.7 & 64.25 & 1.04 & $26.7(31.1)$ & MR & 1.6 & 51.36 & 1.70 \\
\hline P 16 & 86.7 (68.6) & S & 2.8 & 65.88 & 0.97 & $20.0(26.6)$ & MR & 1.3 & 45.90 & 1.58 \\
\hline P 17 & $73.3(58.9)$ & S & 2.7 & 64.25 & 1.03 & $33.3(35.2)$ & MR & 1.9 & 56.54 & 1.67 \\
\hline P 18 & $53.3(46.9)$ & S & 2.3 & 57.69 & 0.85 & $26.7(31.1)$ & MR & 1.6 & 51.36 & 1.39 \\
\hline P 2 & $73.3(58.9)$ & S & 2.4 & 59.33 & 0.90 & $33.3(35.2)$ & MR & 1.7 & 53.18 & 1.46 \\
\hline P 20 & $60.0(50.8)$ & $S$ & 2.3 & 57.69 & 0.56 & $40.0(39.2)$ & MR & 2.1 & 60.46 & 0.92 \\
\hline P 21 & $73.3(58.9)$ & S & 2.7 & 64.25 & 0.72 & $26.7(31.1)$ & MR & 1.7 & 53.18 & 1.18 \\
\hline P 22 & $80.0(63.4)$ & $S$ & 2.7 & 64.25 & 0.61 & $46.7(43.1)$ & MR & 2.1 & 60.46 & 0.99 \\
\hline P 23 & $80.0(63.4)$ & S & 2.7 & 64.25 & 0.83 & $20.0(26.6)$ & MR & 1.5 & 49.54 & 1.36 \\
\hline P 3 & $93.3(75.0)$ & S & 3.0 & 69.16 & 0.87 & $13.3(21.4)$ & $\mathrm{R}$ & 1.2 & 44.08 & 1.42 \\
\hline P 4 & $73.3(58.9)$ & S & 2.7 & 64.25 & 1.01 & $20.0(26.6)$ & MR & 1.5 & 49.54 & 1.64 \\
\hline P 5 & $100.0(84.7)$ & S & 3.0 & 69.16 & 0.60 & $26.7(31.1)$ & MR & 1.7 & 53.18 & 1.03 \\
\hline P 6 & $80.0(63.4)$ & S & 2.7 & 64.25 & 0.89 & $13.3(21.4)$ & $\mathrm{R}$ & 1.3 & 45.90 & 1.45 \\
\hline P 7 & $100.0(84.7)$ & S & 3.3 & 74.07 & 0.85 & $26.7(31.1)$ & MR & 1.6 & 51.36 & 1.39 \\
\hline P 8 & $100.0(84.7)$ & S & 3.3 & 74.07 & 0.94 & $20.0(26.6)$ & MR & 1.3 & 45.90 & 1.53 \\
\hline P 9 & $80.0(63.4)$ & S & 2.7 & 64.25 & 0.89 & $20.0(26.6)$ & MR & 1.6 & 51.36 & 1.45 \\
\hline $\begin{array}{l}\text { PI } 533831 \\
\text { (Sudan) }\end{array}$ & $6.7(15.0)$ & $\mathrm{R}$ & 1.1 & 38.04 & 1.82 & $6.7(15.0)$ & $\mathrm{R}$ & 1.1 & 42.26 & 2.10 \\
\hline PI 655990 & $46.7(43.1)$ & MR & 2.2 & 56.06 & 1.37 & $40.0(39.2)$ & MR & 1.7 & 53.18 & 1.92 \\
\hline PI 656010 & $93.3(75.0)$ & S & 2.5 & 60.97 & 1.27 & $46.7(43.1)$ & MR & 1.7 & 53.18 & 1.66 \\
\hline $\begin{array}{l}\text { PI 656061 } \\
\text { (QL3-India) }\end{array}$ & $13.3(21.4)$ & $\mathrm{R}$ & 1.3 & 41.31 & 1.91 & $0.0(5.2)$ & $\mathrm{R}$ & 1.0 & 40.44 & 2.32 \\
\hline PI 669760 & $66.7(54.8)$ & S & 2.2 & 56.06 & 1.48 & $46.7(43.1)$ & MR & 1.7 & 53.18 & 1.72 \\
\hline $\begin{array}{l}\text { Sekedo } \\
\text { (E525ht.Red) }\end{array}$ & $60.0(50.8)$ & $S$ & 2.5 & 60.97 & 1.48 & $26.7(31.1)$ & MR & 1.5 & 49.54 & 1.97 \\
\hline SESO 1 & $73.3(58.9)$ & S & 2.5 & 60.97 & 1.60 & $26.7(31.1)$ & MR & 1.6 & 51.36 & 1.83 \\
\hline W 3 & $80.0(63.4)$ & S & 3.2 & 72.44 & 0.66 & $13.3(21.4)$ & $\mathrm{R}$ & 1.3 & 45.90 & 1.43 \\
\hline Mean & 80.6 & 2.8 & 65.11 & 0.92 & & 30.1 & 1.6 & 51.29 & 1.52 & \\
\hline $\operatorname{LSD}(0.05)$ & 30.98 & 0.82 & 4.06 & 0.68 & & 31.44 & 0.56 & 2.87 & 0.21 & \\
\hline $\mathrm{CV} \%$ & 23.9 & 18.6 & 3.5 & 45.7 & & 64.9 & 21.9 & 3.5 & 8.40 & \\
\hline
\end{tabular}

Note: PDI = plant disease incidence; values in parenthesis = angle transformed values; $\mathrm{R}=$ resistant $(<20 \%$ PDI $)$; MR = moderately resistant (20-50\% PDI); $\mathrm{S}=>50 \%$ PDI; PDS = plant disease severity; $a=$ disease incidence score at 77 days after planting; $b=$ disease severity score at 77 days after planting; AUDPC = area under disease progress curve. 
Table 3: Correlation coefficient (r) for disease incidence, AUDPC, yield and yield components for sorghum genotypes under natural SDM infection.

\begin{tabular}{|c|c|c|c|c|c|c|}
\hline Variable & $\begin{array}{c}\text { Disease } \\
\text { incidence }(\%)\end{array}$ & AUDPC & $\begin{array}{c}\text { Days to 50 \% } \\
\text { flowering (days) }\end{array}$ & $\begin{array}{l}\text { Plant height } \\
(\mathrm{cm})\end{array}$ & $\begin{array}{c}1000 \text { seed } \\
\text { weight }(\mathrm{gm})\end{array}$ & $\begin{array}{c}\text { Grain yield } \\
\text { (t/ha) }\end{array}$ \\
\hline Disease incidence (\%) & - & & & & & \\
\hline AUDPC & $0.835 * * *$ & - & & & & \\
\hline Days to $50 \%$ flowering (days) & $-0.302 * * *$ & $-0.359 * * *$ & - & & & \\
\hline $\begin{array}{l}\text { Plant height }(\mathrm{cm}) \\
\end{array}$ & 0.014 NS & $-0.003^{\mathrm{NS}}$ & $0.051^{\mathrm{NS}}$ & - & & \\
\hline 1000 seed weight (gm) & $-0.471 * * *$ & $-0.541 * * *$ & $0.193 * * *$ & $0.036^{\mathrm{NS}}$ & - & \\
\hline Grain yield (t/ha) & $-0.585^{* * *}$ & $-0.640 * * *$ & $0.098^{*}$ & $-0.054^{\mathrm{NS}}$ & $0.409 * * *$ & - \\
\hline
\end{tabular}

Downy mildew disease incidence (PDI) was significantly $(\mathrm{P}<0.001)$ correlated with AUDPC $(0.835)$ suggesting that the severity of downy mildew increased with disease incidence. This association was apparent because $P$. sorghi is known to be an obligate biotroph which establishes a prolonged feeding relationship with the living cells of its host, thereby aggravating injury progressive after infection (Perumal et al. 2008). These results were similar to the findings of Kumi et al. (2018a), which reported a significant correlation between SDM disease incidence and severity in sorghum. Downy mildew disease incidence and AUDPC both recorded a significant $(\mathrm{P}<0.001)$ negative correlation coefficient of- 0.585 and0.640 , respectively with grain yield which meant that increased disease incidence and severity of downy mildew resulted in decreased in resultant grain yield of sorghum. Correlation results further showed a significant $(\mathrm{P}<0.001)$ negative correlation for downy mildew disease incidence with days to $50 \%$ flowering (-0.302), and 1000 seed weight (-0.471). Grain yield showed a significant $(\mathrm{P}<$ $0.001)$ positive correlation with days to $50 \%$ flowering (0.098) and 1000 seed weight (0.409). The results in this study suggested that downy mildew disease incidence and AUDPC were negatively and significantly associated with grain yield, days to flowering, and 1000 seed weight, suggesting that genotypes that contribute to reduced SDM incidence and AUDPC would contribute to higher yield and yield-related traits and would, therefore, serve as potential donor parents for breeding sorghum cultivars which are resistant to SMD disease. Similar correlation results were reported in El Salvador (Wall et al. 1992), Nigeria (Anaso et al. 1989) and U.S.A (Craig et al. 1989).

In conclusion, significant phenotypic variations in traits were observed among the 100 sorghum genotypes in response to downy mildew disease reaction and grain yield. Two genotypes PI 533831 (Sudan) and PI 656061 (QL3India) were found to be resistant and high yielding at both locations and could be utilized in a sorghum program to breed for resistance to Peronosclerospora sorghi.

\section{ACKNOWLEDGEMENTS}

This research was funded by the Carnegie Cooperation of New York through the Regional Universities Forum for Capacity Building in Agriculture (RUFORUM). The authors are grateful to the National Semi-Arid Resources Research Institute (NaSARRI) Sorghum project and the United States Department of Agriculture-Agricultural Research Service (USDA-ARS).

\section{REFERENCES}

Aegerter B J, Nuñez JJ, Davis RM. 2003. Environmental factors affecting rose downy mildew and development of a forecasting model for a nursery production system. Plant Dis 87: 732-738.

Anaso AB, Emechebe AM, Tyagi PD, Manzo SK. 1989. Assessment of loss in yield due to sorghum downy mildew (Peronosclerospora sorghi) of maize in Nigerian guinea savanna. Trop Pest Manag 35 (3): 301-303.

Barbosa FCR, Casela CR, Pfenning L H, Santos FG. 2004. Identification of sources of resistance in sorghum to Peronosclerospora sorghi. Fitopatologia Brasileira 30 (5): 522-524.

Bigirwa G, Adipala E, Esele JP. 1998. Occurrence of Peronosclerospora sorghi in Uganda. Plant Disease, 82 (7): 757-760.

Bigirwa G, Adipala E, Esele JP, Cardwell KF. 2000. Reaction of maize, sorghum and Johnson grass to Peronosclerospora sorghi. Intl J Pest Manag 46 (1): 1-6.

Bock CH, Jeger M, Mughogo L, Cardwell K, Mtisi E, Kaula G, Mukansbima D. 2000. Variability of Peronosclerospora sorghi isolates from different geographic locations and hosts in Africa. Mycol Res 104: 61-68.

Cohen Y, Sherman Y. 1977. The role of airborne conidia in epiphytotic of Sclerospora sorghi on sweet corn. Phytopathology 67 (4): 515-521.

Craig J, Odvody GN, Wall GC, Meckenstock DH. 1989. Sorghum downy mildew loss assessment with near-isogenic sorghum populations. Phytopathology 79: 448-451.

FAOSTAT (Food and Agriculture Organization of the United Nations). 2013. Production statistics: crops. FAO Statistical Databases, FAO, Rome.

Frederiksen RA. 2000. Diseases and disease management in sorghum. In: Smith CW, Frederiksen RA (eds). Sorghum: Origin, History, Technology, and Production. John Wiley and Sons, Inc., New York, NY.

Isakeit T, Odvody G, Jahn R, Deconini L. 2003. Peronosclerospora sorghi resistant to metalaxyl treatment of sorghum seed in Texas. Phytopathology 93: S39.

Jeger, MJ, Gilijamsea E, Bock, CH, Frinkinga HD. 1998. The epidemiology, variability and control of the downy mildew of pearl millet and sorghum, with particular reference to Africa. Plant Pathol 47: 544-569.

Kamala V, Bramel PJ, Sivaramakrishnan S, Chandra S, Kannan, S, Harikrishna S, Rao DM. 2006. Genetic and phenotypic diversity in downy-mildew-resistant sorghum (Sorghum bicolor (L.) Moench) germplasm. Genet Res Crop Evol 53 (6): 1243-1253. DOI: 10.1007/s10722-005-5678-7

Kamala V, Singh SD, Bramel PJ, Manohar Rao D. 2002. Sources of resistance to sorghum downy mildew in wild and weedy sorghums. Crop Sci 42: 1357-1360. DOI: 10.2135/cropsci2002.1357. 
Kumi F, Agbahoungba S, Badji A, Mwila N, Ibanda A, Anokye M, et al. 2018b. Genetic diversity and population structure of Peronosclerospora sorghi isolates of Sorghum in Uganda. Intl Environ Agric Biotechnol 3 (5): 1656-1667. DOI: 10.22161/ijeab/3.5.11

Kumi F, Agbahoungba S, Badji A, Odong T, Edema R, OchwoSsemakula M, et al. 2018a. Incidence and distribution of downy mildew disease (Peronosclerospora sorghi) of sorghum in Uganda. Intl J Adv Res 6 (5): 954-965. DOI: 10.21474/IJAR01/7117

Miller FR, Dusek TF, Prihoda KL, Rooney LW. 1992. Registration of RT436 sorghum parental line. Crop Sci 32 (6): 1518.

Pande S, Bock CH, Bandopadhyay R, Narayana YD, Reddy BVS, Lenne JM, Jeger MJ. 1997. Downy mildew of sorghum. Information bulletin No. 51. International Crops Research Institute for the Semi-Arid Tropics (ICRISAT), Patancheru, India.

Payne RW, Murray DA, Harding SA, Baird DB, Soutar DM, Lane P. 2009. GenStat for Windows, 12th ed. VSN International, Hertfordshire, UK.

Perumal R, Nimmakayala P, Erattaimuthu SR, Reddy UK, Prom LK, et al 2008. Simple sequence repeat markers useful for sorghum downy mildew (Peronosclerospora sorghi) and related species. BMC Genetics 9: 1-14. DOI: 10.1186/1471-2156-9-77.

Prom LK, Erpelding JE, Montes-Garcia N. 2007. Chinese sorghum germplasm evaluated for resistance to downy mildew and anthracnose. Commun Biometry Crop Sci 2 (1): 26-31.

Prom LK, Montes-Garcia N, Erpelding JE, Perumal R, Medina-Ocegueda S. 2010. Response of sorghum accessions from Chad and Uganda to natural infection by the downy mildew pathogen, Peronosclerospora sorghi in Mexico and the USA. J Plant Dis Protect 117 (1): 2-8. DOI: 10.1007/bf03356326

Prom LK, Perumal R, Montes-Garcia N, Isakeit T, Odvody GN, et al 2014. Evaluation of Gambian and Malian sorghum germplasm against downy mildew pathogen, Peronosclerospora sorghi, in Mexico and the USA. J General Plant Pathol 81 (1): 24-31. DOI: 10.1007/s10327014-0557-8
Radwan GL, Perumal R, Isakeit, T, Magill CW, Prom LK, Little CR 2011. Screening exotic sorghum germplasm, hybrids, and elite lines for resistance to a new virulent pathotype (P6) of Peronosclerospora sorghi causing downy mildew. Plant Health Progress. DOI: 10.1094/PHP-2011-0323-01-RS.

Rao PS, Kumar CG. 2013. Characterization of Improved Sweet Sorghum Cultivars. Springer Briefs in Agriculture. DOI: 10.1007/978-81-3220783-2

Shaarawy MA, Abdel-Monem AM, El-Waki AA. 2002. A modified method for detecting oospores of Peronosclerospora sorghi, the causal organism of sorghum downy mildew in sudan grass seeds (Sorghum sudanense). Egyptian J Agric Res 80 (3): 1033-1043.

Simko I, Piepho HP. 2012. The area under the disease progress stairs: Calculation, advantage, and application. Phytopathology 102: 381389.

Sokal RR, Rohlf FJ. 1995. Biometry. 3rd Edition. Freeman, New York, USA.

Tuleen DM, Frederiksen RA, Vudhivanich P. 1980. Cultural practices and the incidence of sorghum downy mildew in grain sorghum. Phytopathology 70: 905-908.

Wall GC, Frederiksen RA, Craig JD, Jeger MJ. 1992. Epidemiology of sorghum diseases in Central America: A case study. In: de Millian WAJ, Frederiksen RA, Bengston GD. (eds.) Proceedings, Sorghum and Millets Diseases, A Second World Review. ICRISAT, Pataacheru, Andhra, Pradesh, India.

Ward JMJ, Laing MD, Rijkenberg FHJ. 1997. Determining incidence and severity of downy mildew disease in Maize and Sorghum. Plant Dis 81: 41-48.

Wasake ED. 2016. Sorghum growing in Uganda: beer and heaven? INACHEE, Uganda.

William HA, Darrell JC, Girish B. 1990. Use of the Arcsine and Square Root Transformations for Subjectively Determined Percentage Data. Weed Sci 38 (4/5): 452-458 